\title{
CEROUS MAGNESIUM NITRATE: A MAGNETIC TEMPERATURE SCALE $0.002-2^{\circ} \mathrm{K}$
}

\author{
R.P. HUDSON and R.S. KAESER \\ National Bureau of Standards, Washington, D. C. \\ (Received 18 July 1966 )
}

\begin{abstract}
Isentropic demagnetizations from known starting conditions yielded a determination of the entropy-magnetic susceptibility, or $S-x$, relation for single-crystal spheroids of the paramagnetic salt cerous magnesium nitrate, CMN. Enthalpy differences, $\Delta Q$, between the low temperature end-points thus reached and an (arbitrary) reference temperature near $1^{\circ} \mathrm{K}$ (determined by gamma ray heating) yielded a corresponding $Q-S$ curve, whence one obtained $S-T$ and $X-T$ relations, $T$ being the thermodynamic temperature. The salt exhibits a broad maximum in $x$ centered on $S / R=0.32$, for which region the absolute temperature is determined to be $0.0015^{\circ} \mathrm{K}$. Our results disagree significantly with those of other workers (even $S-X$, which is not subject to large experimental error) save for the observation that CMNobeys the Curie law at least down to $0.006^{\circ} \mathrm{K}$.
\end{abstract}

At high temperatures $\left(T>0.006^{\circ} \mathrm{K}\right)$, all specimens show a heat capacity $C / R=$ $b T^{-2}$ with $b=5.76 \times 10^{-6} \mathrm{deg}^{2}$. Superimposed upon this is a small "anomaly" first appearing at approximately $0.015^{\circ} \mathrm{K}$ and reaching a maximum near $0.025^{\circ} \mathrm{K}$. The attribution of such an effect to small concentrations of magnetic impurities cannot be reconciled with the results of chemical analysis. Other evidence, moreover, supports the idea of a non-magnetic origin for this anomaly.

\section{Introduction}

SINCE first studied as a magnetic cooling and thermometric medium [1], the paramagnetic salt cerous magnesium nitrate, $2 \mathrm{Ce}\left(\mathrm{NO}_{3}\right)_{3} \cdot 3 \mathrm{Mg}\left(\mathrm{NO}_{3}\right)_{2} \cdot 24 \mathrm{H}_{2} \mathrm{O}$ (hereinafter referred to as CMN), has stood pre-eminent as a working substance for the adiabatic demagnetization region [2]. Owing to the high magnetic dilution, the paramagnetic susceptibility follows the Curie Law down to approximately $0.006^{\circ} \mathrm{K}$ (highly convenient for thermometric indication) and can be used to produce temperatures a factor of at least two, and probably considerably more [3], lower. In the ground, $J=5 / 2$, manifold the first excited doublet lies some 34 deg above the ground doublet [4] and the Curie Law behavior is exhibited through and beyond the liquid helium region $[5,6]$.

As the applications of this salt multiplied and the question of reliability of the temperature scale provided by the first rough measurements aroused increasing interest, it became evident that new determinations were highly desirable. A series of these was undertaken [3], the first of which indicated that below the Curie Law region the absolute temperatures were 
considerably lower than suggested by the pioneer measurements and, furthermore, that the value of $b$ in the "high" temperature approximation for the specific heat $C$, viz. $C / R=b / T^{2}$, was als much smaller than the published value.

Because the first experiments were carried out on a roughly ellipsoidally-shaped specimen and the above first check was made on a sphere, we next studied an ellipsoid and obtained [3] an entropy-temperature curve intermediate between the first two. The two new studies were reported in graphical form only, since we regarded them as preliminary, anticipating data of higher quality made on a new sphere with improvements in the apparatus and experimental procedures. The latter are the subject of this paper; which is being published now (a) because it is long overdue, (b) because other groups have recently reported $[7,8]$ on a quite different approach to the determination of the CMN temperature scale with results tending to reinforce our preliminary findings, and (c) due to further enhanced interest in the scale with respect to recent important experiments, utilizing CMN, investigating the possible existence of a super fluid transition below $0.01^{\circ} \mathrm{K}$ in liquid $\mathrm{He}^{3}$ [9].

\section{Experimental Method}

The approach adopted was the classical magnetic cooling and gamma-ray heating one [10] and, in detail, followed closely that described in earlier experiments by Ambler and Hudson [11] on a different paramagnetic salt. The CMN specimen was adiabatically demagnetized from known start ing conditions of field, $H_{i}$, temperature, $T_{i}$, and hence entropy, $S$ (calculated using the known $g$-value, $1.84[12]$ ) to a measured final susceptibility, $X_{0}$ and hence magnetic temperature, $T \otimes$ (defined by $T \otimes=c / X$, where $c$ is the Curie constant, 0.318 e.m.u./g ion). In this way a complet $S-T \otimes$ curve was obtained and combined with a heat content, $Q-T \otimes$ curve established via the "total-Q" gamma-ray heating method $[1,11]$ to yield the $S-T$ and $T \otimes-T$ relations.

The maximum magnetic field available was 2.3 tesla ( 1 tesla $=10^{4}$ gauss) and the lowest valu employed was 0.176 tesla; even the latter, applied at a starting temperature of, typically, $1.13^{\circ} \mathrm{K}$, produces a very low final temperature, viz. $0.035^{\circ} \mathrm{K}$, wherefore weak-field demagnetizations were also performed from starting temperatures up to as high as $2.08^{\circ} \mathrm{K}$ in an attempt to span the higher reaches of the $S-T \otimes$ curve.

\section{Apparatus}

(a) Specimens and method of mounting.

The specimens were ground to the desired shape from large single crystals of CMN. The constituent cerous nitrate was the purest commercially available and the CMN was recrystallized several times before final growth was initiated. Upon analysis by "a general spectrochemical method" the identifiable impurities were reported [13] to be: calcium and copper - traces (indicating $10^{-6}$ to $10^{-5}$ ); silicon and neodymium - very weak (indicating $10^{-5}$ to $10^{-4}$ ).

Although the predominant $\mathrm{Ce}^{3+}$ tends to obscure other rare earth spectra, it was felt that the evidence was strongly in favor of all undesirable (magnetic) impurities being present in insignificant amounts, or at concentrations so small that the effects would be confined to a very small hfs entropy contribution above $0.05^{\circ} \mathrm{K}$, readily identifiable if present and only necessitating at most a very small correction to the "initial entropy" (see $4 \mathrm{~d}(\mathrm{i}$ ) and (ii) 
elow and Appendices).

The specimen was mounted within a glass vacuum case at the top of a $10.5 \mathrm{~cm}$ long lowhermally-conducting support, anchored at its lower end into the center of the ground-glass lug which sealed off the vacuum case. The spherical spcimens were either lashed with cotton hread to a small glass cup fixed to the top of a paper tube (actually a drinking "straw") or mpaled by means of a vertical axial hole on the end of a drawn glass spill narrowed to a 2.5 m long $0.084 \mathrm{~cm}$ o.d. section at the top; the ellipsoid was glued and tied to the slit and anned-open ends of a paper tube.

The $c$-axis of the crystal coincides with the $g=$ direction. In the plane perpendicular to his, two mutually perpendicular axes were marked, the one to be set vertically for the susceptipility measurement and the other in the direction of the field of the electromagnet. The marking ras actually accomplished by drilling holes with a $0.064 \mathrm{~cm}$ drill; one of these was enlarged blightly and used to mount sphere $I$, as described above.

Owing to the marked anisotropy of CMN, a crystal will tend to line up with the magnetic field direction automatically. In assembling the apparatus visual alignment was used, good to probably $\pm 5^{\circ}$. This could be checked, and corrected if necessary, by generating a small field and measurIng the susceptibility as the magnet was rotated over a few degrees. The demagnetization data were reproducible enough, too, to observe the effects of displacements of $H$ from $g{ }_{\perp}$ by $5^{\circ}$. Noting that cosine $5^{\circ}$ is 0.9962 , one sees that even a $5^{\circ}$ error would reduce the effective field by only 0.4 per cent, but the above checks permitted a considerable improvement over this.

As the inside diameter of the vacuum case was only $30 \mathrm{~mm}$ it was necessary to be sure that the $25-\mathrm{mm}$ diameter spherical specimens were centrally placed (sphere I was actually $27 \mathrm{~mm}$ dia.). This was confirmed by taking $\mathrm{X}$-ray photographs after assembly but prior to mounting the dewars.

\section{(b) Susceptibility measurement}

The CMN specimen formed the core of a mutual inductance which could be measured by means of a Hartshorn bridge [14], normally operated at $267 \mathrm{H}$. The secondary, comprising a pick-up winding (1400 turns of 43 s.w.g. copper wire) plus symmetrically disposed bucking coils, was wound directly on the vacuum jacket; the primary, one layer of $30 \mathrm{s.w.g.,} \mathrm{was} \mathrm{wound} \mathrm{directly} \mathrm{on} \mathrm{to} \mathrm{the}$ tail section of the surrounding liquid helium dewar and immersed in the liquid nitrogen contained in the exterior dewar.

Calibration of the magnetic thermometer was carried out between 1.1 and $4.2^{\circ} \mathrm{K}$, deriving the temperature from the vapor-pressure of the liquid helium with corrections for hydrostatic head in the $\mathrm{He}^{4} \mathrm{I}$ region. The ensuing relationship between bridge turns, $n$, and temperature was the customary

$$
n^{\prime}=n-n_{\infty}=A T^{-1}
$$

with $A=3$ deg approximately and $n_{\infty}$, the departure from exact bucking of mutual inductance, about 20. One bridge turn corresponds to $3.06 \mu \mathrm{H}$. With such an arrangement the maximum susceptibility encountered corresponded to $n \approx 900$, comfortably inside the $n=1111.1$ range of the bridge. The minimum detectable bridge unbalance was 0.002 turn at $25 \mathrm{~mA}$ primary current (equivalent primary field $\approx 0.75 \times 10^{-4}$ tesla) which corresponds to $\pm 0.01^{\circ}$ at $4^{\circ} \mathrm{K}, \pm 0.003^{\circ}$ at $2^{\circ} \mathrm{K}$, and $0.0008^{\circ}$ at $1.1^{\circ} \mathrm{K}$. 


\section{(c) Field and temperature controls}

High stability of both magnetic field and temperature are required for accurate characterization of the (entropy) state of the CMN in an adiabatic demagnetization, while temperature control is of extreme importance during the calibration. The $100 \mathrm{~kW}$ electromagnet was excited by powers ranging from $50 \mathrm{~W}$ up to the maximum of the available $25 \mathrm{~kW}$ generator and adequate field stability was achieved by current stabilization, obtained by means of a shunt-tapping electronic servo-loop in the exciter circuit of the generator. The magnet current was stabilize against drift to $1: 10^{4}$. The magnet was calibrated against the level-set control reading by means of a rotating-coil gaussmeter. This relatively crude instrument limited the accuracy of measurement of $H$ to about $\pm 1 / 4$ per cent for $H$ greater than about 0.8 tesla. Below this, the non-reproducibility of the $H$ vs. $I$ characteristic due to remanence degraded the accuracy to about \pm 0.002 tesla.

The helium vapor-pressure was measured, according to its magnitude, by a manometer or a McLeod gauge. A line tapped into the cryostat at its head led to a small mercury manometer in a photocell amplifier unit which in turn controlled a needle valve in the main pumping line. With this device, temperature drift was kept negligibly small. Magnetization temperatures usually ranged between the vapor pressure equivalents 0.3 and $0.6 \mathrm{~mm} \mathrm{Hg}$, the vacuum pump now operating via a fully-open 3-in. gate valve. During the magnetization period of some 6-8 min the McLeod reading might change by as much as $0.03 \mathrm{~mm} \mathrm{Hg}$ at $0.4 \mathrm{~mm}$, or $\Delta T=0.01^{\circ}$ at $1.14^{\circ} \mathrm{K}$; usually the temperature drift was much less than this.

\section{(d) Gamma-ray heating unit.}

For heating the salt after cooling to low temperatures, the cryostat was swung out of the electromagnet. A massive lead pig located directly beneath contained a nominal $250 \mathrm{mCi} \mathrm{Co}^{60}$ source comprising 16 cobalt wires placed vertically and axially-symmetrically in a cylindrical brass container. This source could be raised from a remote position to the level of the salt in about 2 sec. Using this arrangement, a one-inch diameter CMN sphere could be heated at approximately $2000 \mathrm{ergs} / \mathrm{min}$, which is about 50 times larger than the natural heat leak.

The gamma heating was not initiated instantaneously, of course. To investigate the detailed behavior we substituted a modified photomultiplier tube for the cryostat and fed the output signal to a chart recorder. From these observations we found that the elevation period was equivalent to about $0.2 \mathrm{sec}$ of full gamma heating.

\section{Results}

\section{(a) General}

All three specimens studied showed the characteristic CMN behavior: dramatic cooling for even small entropy reduction (indicative of the very weak ion-ion interaction); the entropy varying as $(T \otimes)^{-2}$ down to about $0.006^{\circ}$ [15] (but see $4 \mathrm{~d}(\mathrm{i})$ for anomalous features); practically constant susceptibility, or $T^{\otimes}$, for all entropies smaller than, 0.38R; pseudo specific heat $C^{\otimes},=d Q / d T^{\otimes}$, varying as $\left(T^{\otimes}\right)^{-2}$ for magnetic temperatures greater than $0.006^{\circ}$ (but see $4 \mathrm{~d}(\mathrm{ii})$ for anomalous behavior at "high" temperatures).

The latter property is indicated by the susceptibility decreasing linearly with time under 
onstant heating, as follows from combining the relations $C\left(T^{\otimes}\right)^{2}=$ const. and $x^{\otimes}=$ const. , irst noted by Cooke and Hull [16]. Furthermore, as long as both $C^{\otimes}$ and $S$ manifest an inverse quare dependence on $T \otimes$, the latter is identical with $T$, the absolute temperature (so long as $\otimes$ is meaningful, i.e. $T$ is uniform throughout the specimen).

b) Entropy-magnetic temperature, $S-T \otimes$, relation

Within the limits of experimental error, the three different specimens gave identical $S-T \otimes$ urves, as shown in Fig. 1 which is actually a plot of $S-X$ and is better suited to high $X$ (low (®) values.

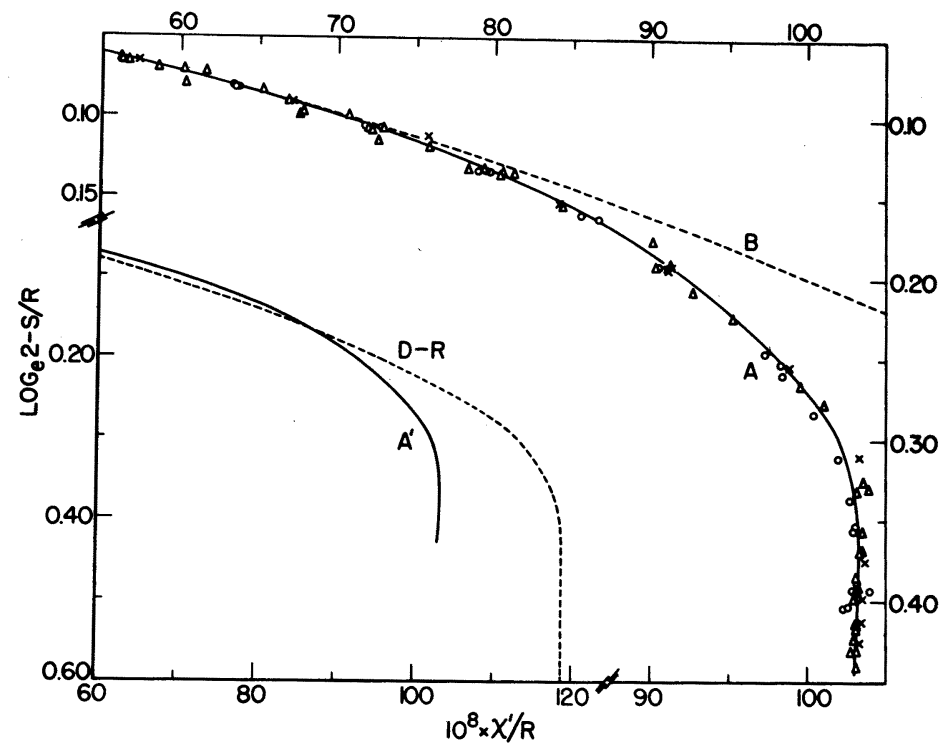

FIGURE 1

Entropy vs. susceptibility (per mole, divided by the gas constant). $0 . X, \Delta, \longrightarrow$ data for sphere I, "ellipsoid", and sphere II, respectively. Curve $A$ is a smooth line drawn through these points and curve $B$ corresponds to $\ln 2-S / R=2.88 \times 10^{-6}\left[T \otimes T^{-2}\right.$. Inset, comparison with results of Daniels and Robinson [1], curve $D-R$, curve $A^{\prime}$ being a reproduction of curve $A$.

(The susceptibility of the ellipsoid was reduced to the case of a sphere by applying the appropriate shape-dependent demagnetizing correction, $\Delta$, to the magnetic temperature, $T^{*}$, obtained from the formula $T^{*}=c / X$, since $T^{\otimes}=T^{*}+\Delta$ [17]. Using the latter and the relation In $2-S / R=\frac{1}{2} b\left(T^{\otimes}\right)^{-2}$, one may obtain the value of $\Delta$ from the negative intercept on the abscissa axis of a plot of $(S / R)^{-1 / 2}$ vs. $T^{*}[1]$. The result was $\Delta=1.75 \times 10^{-3}$ deg, and from the slope we calculated $b=5.76 \times 10^{-6} \mathrm{deg}^{2}$.)

To obtain the value of $b$ from the three sets of data, we plotted $\ln 2-S / R$ vs. $\left(T^{\otimes}\right)^{-2}$, as shown in Fig. 2. 


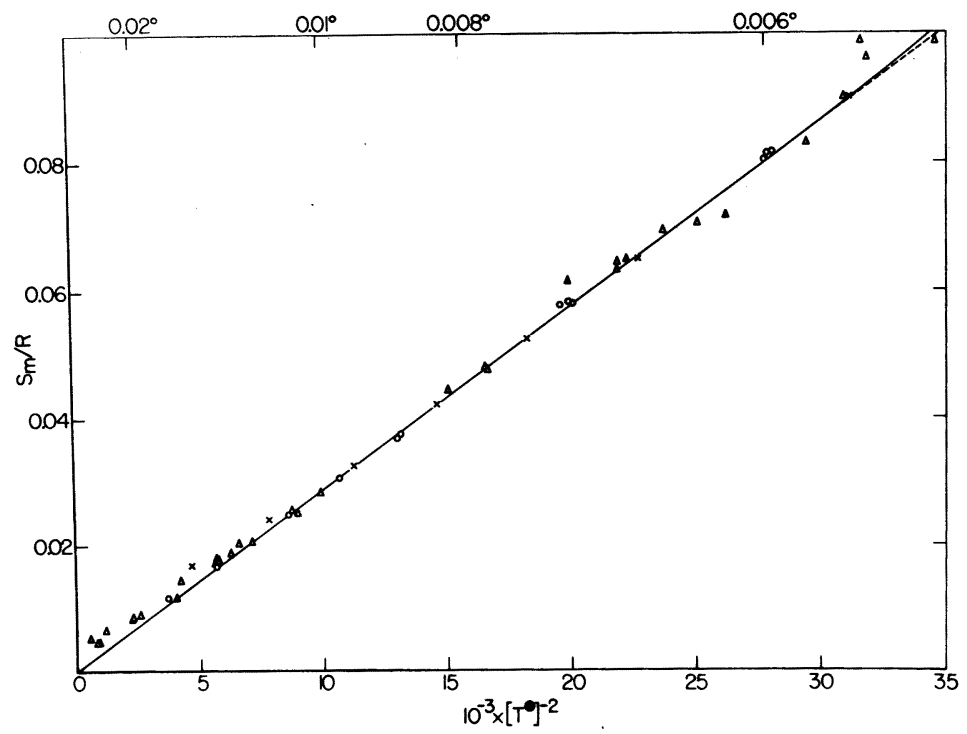

FIGURE 2

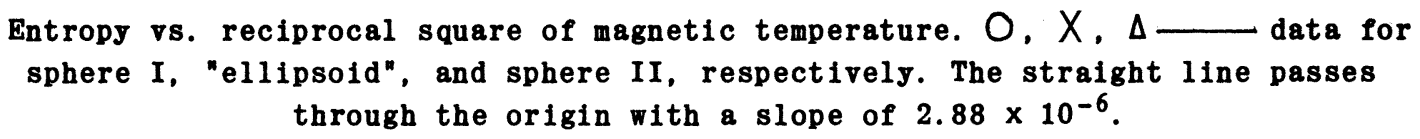

Two points need to be emphasized immediately, viz. the entropy, $S$, here is that of the spin system alone, calculated using $g=1.84$ and the appropriate values of $H$ and $T$; and, as is evident from Fig. 2, deviations from linearity occur at the high temperature end, $T^{\otimes}>0.015^{\circ}$. Deferring further discussion of these points until $4 \mathrm{~d}(i)$ below, we note that the straight line drawn through the mid-region points of Fig. 2 and through the origin has a slope of $2.88 \times 10^{-6}$, corresponding to $b=5.76 \times 10^{-6} \mathrm{deg}^{2}$ as already found by the alternative procedure applied to the ellipsoid data (above). Again, one line represents the data for all specimens equally well.

(c) Heat content - susceptibility, Q-X, relation

For a given entropy, the heat content was determined by measuring the time required to warm the specimen up from the low temperature reached upon adiabatic demagnetization to a reference temperature sufficiently high as to guarantee uniformity of temperature distribution. The latter should be indicated by the susceptibility vs. time curves being independent of total heating time. At very low temperatures the thermal diffusivity is not high enough to counter the inhomogeneity of the gamma ray heating and in that region the lower the initial entropy, the further the warm-up curve departs from the equilibrium or "master-Q" curve [11].

The bridge balance, $n$, changed from a maximum of about 900 turns (for the lowest entropies) to about 20 turns at bath temperature. For operational convenience, intermediate balance points were timed for 50 turn intervals above 80 which was the reference reading, corresponding to $T^{\otimes} \approx 0.05^{\circ}$. Between 600 and 80 , the $n$ vs. $t$ plot was linear for the spherical specimen $I$, indicating $C^{\otimes} \propto\left(T^{\otimes}\right)^{-2}$ (see 4 a above). For those demagnetizations which carried $n$ initially beyond 
he linear region, however, the linear portions were not identical. The remainder of the curves ould be used to establish the slope value, which is needed for calibrating the gamma source as heater. In order to be certain that this slope was determined unambiguously, readings for phere II were carried on down to $n=30\left(T^{\otimes} \simeq 0.25^{\circ}\right)$. As a result, a quite unexpected feature ame to light, which is discussed in $4 \mathrm{~d}$ (ii) below.

In using the "total-Q" method, reliance is placed upon the heating rate being so fast that he background heating rate is relatively very small. Then errors due to the difficulty of laking an accurate correction for the "drift" time prior to application of the gamma radiation, nd those due to making the assumption that the "drift" is constant for the duration of the leating period, and from run to run, are minimized. In our case, the ratio of gamma heat to drift" was about $50: 1$. Heating periods ranged from 50 to $110 \mathrm{sec}$ while the "gamma equivalent" of the fore-period ranged from 1 to $3 \mathrm{sec}$. For all but the highest-field demagnetizations, the eating curve could be extrapolated back to the zero-time value of $n$ for a satisfactory drift correction. For the rest, a pronounced initial curvature made the extrapolation somewhat suspect and a check was always made to see if the findings were consistent with the $50: 1$ ratio valid for the higher temperature points. One favorable circumstance is that for a given correction the percentage of total heating time is smallest for the most awkward points.

In Fig. 3 are shown some of the warm-up data for sphere II, where the heat content is plotted along the abscissa in seconds of heating time. If there were no inhomogeneity effect a unique,

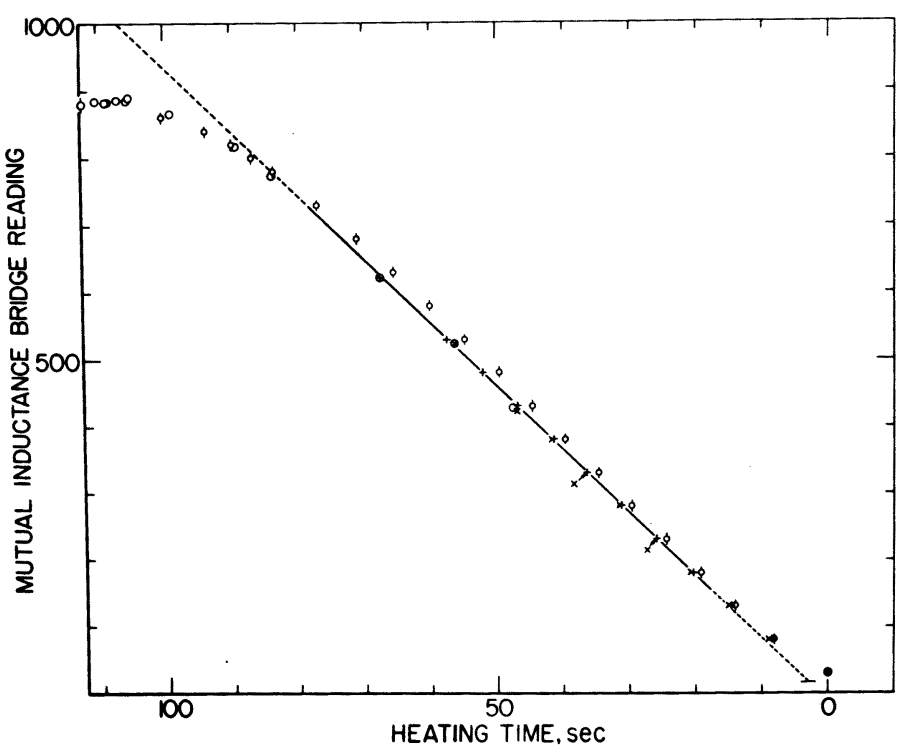

FIGURE 3

Representative data for sphere II of bridge turns $\left(n-n_{\infty}\right)$ vs. time under constant heating. $\otimes, x ; \oplus,+$; initial (extrapolated) reading and subsequent readings for two separate "demagnetizations" within the linear region; $\phi . \phi ;-\operatorname{similar}$ for an extreme cooling; 0 , - initial readings (only) for other warm-up curves; 2. universal end-point at $1^{\circ} \mathrm{K}$. Drawn line represents linear portion of the "master- $Q$ curve", corresponding to homogeneous heating of the crystal. 
master- $Q$, curve would be obtained. We have plotted the extreme point for each entropy, together with sufficient of the intermediate points to illustrate their interpolating usefulness for the higher initial entropies and their deceptive nature otherwise. Note that between approximately 600 turns and 80 turns the $n-t$ characteristic is quite linear.

From the original large-scale plot of Fig. 3 it may be seen that the warm-up curves for the lowest-entropy demagnetizations do not quite coincide with the master curve even at 80 turns on the bridge (63 actual turns, allowing for 17 turns residual coil unbalance). Although the sprea in points is most probably very largely a manifestation of experimental scatter, we decided to choose 30 turns (13 "real turns" or $\sim 0.25^{\circ} \mathrm{K}$ ) for our reference point for heating time.

Referring back to 4 (b) we know that $C T^{2} / R=5.76 \times 10^{-6} \mathrm{deg}^{2}$; and from equation (1), differentiating w.r.t. time,

$$
d n / d t=-A T^{-2} d T / d t
$$

Hence the heating rate is given by

$$
\frac{d}{d t}(Q / R)=(C / R) \frac{d T}{d t}=-\frac{5.76 \times 10^{-6}}{A} \cdot \frac{d n}{d t}
$$

and substituting $-d n / d t=9.43$ turns/sec (from Fig. 3) and $A=3.190 \mathrm{deg}$, one finds

$$
\frac{d}{d t}(Q / R)=1.70 \times 10^{-5} \cdot \mathrm{deg} \mathrm{sec}^{-1}
$$

corresponding to $1830 \mathrm{ergs} / \mathrm{min}$ in the case of sphere II.

\section{(d) Anomalous behavior at high temperatures}

(i) Cooling. In deriving an $S-T \otimes$ curve we calculate $S$ for each point on the assumption that it is the same as it was at the commencement of the demagnetization. At that point the salt is at a high (approx. $1^{\circ} \mathrm{K}$ ) temperature and, in general, the entropy, $S_{i}$, will be given by

$$
S_{i}=R \ln 2+S_{l}-S_{m}
$$

where $S_{l}$ is the entropy due to the lattice and $-S_{m}$ is the reduction in entropy due to the magnetic field.

A value for $S_{l}$ is available from the work of Bailey [18], e.g. about $2 \times 10^{-4} \mathrm{R}$ at $1^{\circ} \mathrm{K}$, and is seen to be negligible for all except the smallest values of $S_{m}$ [19]. In 0.176 tesla at $1.134^{\circ} \mathrm{K}, S_{m}$ and $S_{l}$ are $4.6 \times 10^{-3} R$ and $2.8 \times 10^{-4} R$ respectively.

We thought it would be of interest to make an independent determination of $S_{l}$ by keeping $T_{i}$ considerably higher for some low-field demagnetizations, so that $S_{l}$ could be comparable with $S_{m}$. The results were quite unexpected and indicated a breakdown of the validity of the isentropicity assumption, i.e. the lattice temperature now lags behind that of the electron spin system and the process is no longer thermodynamically reversible.

The effect is quite evident in Fig. 2 where the points towards the origin deviate from the 
straight line. Had we included the term $S_{l} / R$ in calculating the entropy for Fig. 2, the anomalous points could have been lowered, it is true, but by a negligible amount for $1.1^{\circ} \mathrm{K}$ magnetizations. On the other hand, points for magnetizations at $2.1^{\circ} \mathrm{K}$ fall well below the line and indicate unexpectedly low temperatures, which is nonsense.

The fact that the best straight line through the data of Fig. 2 passes through the origin suggests, furthermore, that the lattice is not involved in the cooling process using high fields, 1.e. the spins must cool immediately "out of the lattice's reach" and any ensuing manifestation of the lattice's relatively high temperatures would be indistinguishable from any other kind of heat leak. Accepting this line as "correct", one notes that the observed behavior approximates this more and more closely as $T_{f}$ gets lower. By $0.015^{\circ}$ all anomalous effects have disappeared, independently of the $H_{i}$ and $T_{i}$ values used to reach there. (The trend in the points as $T_{i}$ is lowered is the important feature; the "origin" argument would only be conclusive if there were no experimental scatter. As we shall show later, the entropy correction due to impurities which possess hfs is negligible and the validity of the origin of Fig. 2 as a guide point for drawing the straight line is not compromised thereby.)

(ii) Heating. In Fig. 3 one may notice that the linear dependence of bridge turns upon time (for continuous gamma irradiation), which sets in as $n$ falls below 600 , is not maintained to the very highest temperatures. In fact, anomalous behavior which is barely discernible within the experimental scatter at 130 turns $\left(0.03^{\circ}\right)$ is very marked by 80 turns $\left(0.05^{\circ}\right)$. This slowing down of the rate of warming can only be expla ined, presumably, by the heat capacity rising above that due to the spins alone, which if falling as $T^{-2}$.

Figure 4 shows the high temperature portion of a gamma heating curve, the dotted line indicating the course the data would have taken for a normal $T^{-2}$ specific heat. It must be

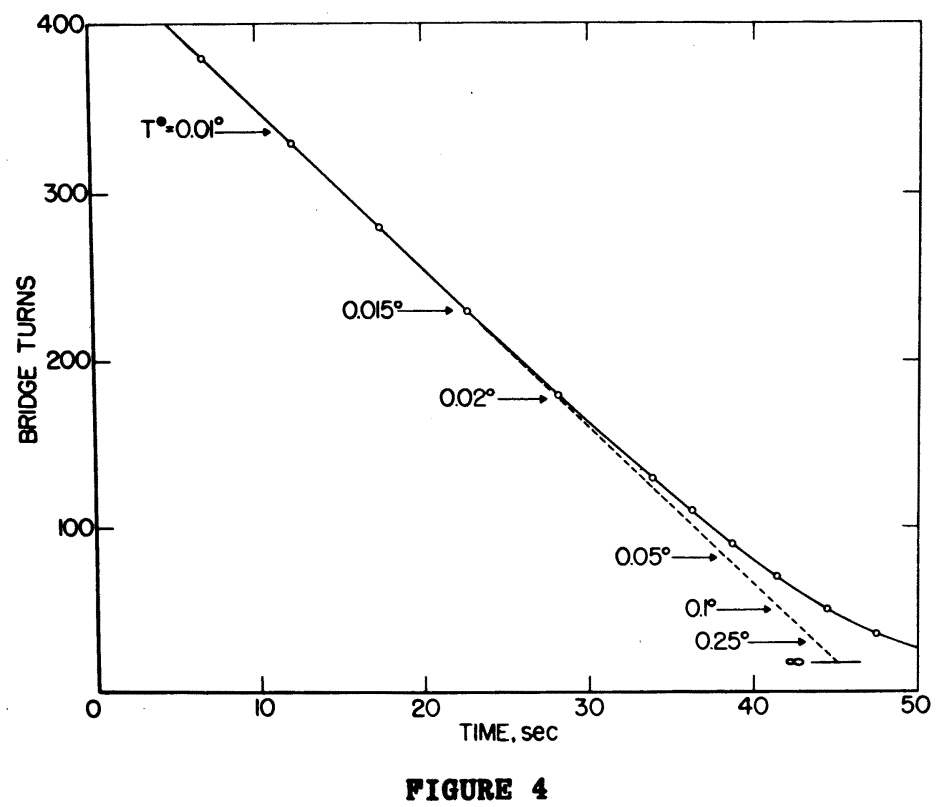

Bridge turns $\left(n-n_{\infty}\right)$ vs. time for high temperature portion of a heating curve. The straight line corresponds to a $T^{-2}$ dependence for the heat capacity and is identical with the straight line in Fig. 3. 
emphasized that the anomalous, or excess, heat capacity is very small and is only made manifest by the extremely small heat capacity of CMN itself (see Appendix 3). The associated entropy amounts to roughly $0.0014 R$, which is to be compared with $R$ ln 2 or $0.6932 R$ for the total CMN spin entropy. (On the other hand, the spin system itself only gains $0.0128 R$ above $0.015^{\circ} \mathrm{K}$.) The surprising fact is its appearance at so low a temperature as $0.015^{\circ} \mathrm{K}$; lattice entropy is very decisively ruled out, if it is permissible to extrapolate the results of calorimetry obtained above $2^{\circ} \mathrm{K}$ [18] or to assume, at least, that the Debye characteristic temperature changes very little below $2^{\circ} \mathrm{K}$.

The ready conclusion that the possible $10^{-4}$ concentration of Nd impurity explained all was easily shown to be erroneous and we resolved upon obtaining a quantitative determination of all the spectroscopically-indicated magnetic impurities. The results (Appendix 1 ) showed that the anomaly cannot be due to these impurities (their associated hfs entropy - see Appendix 2 amounts to $10^{-4} R$ which has a negligible influence on Fig. 2) hence we must conclude - however surprised - that the anomaly is non-magnetic in origin. The concept of such a "ballast" is also consistent with the postulated non-isentropic nature of weak-field demagnetizations raised in the discussion of the features of Fig. 2, above.

\section{(e) Absolute temperatures}

Using the value of $\dot{Q} / R$ from equation (4), section $4(\mathrm{c})$, and the measured time to heat from the (extrapolated) initial low temperature point to the high reference temperature, values of $Q / R$ were calculated. These were plotted against the corresponding entropy, $S / R$, values calculated for the initial $H$ and $T$, as shown in Fig. 5 .

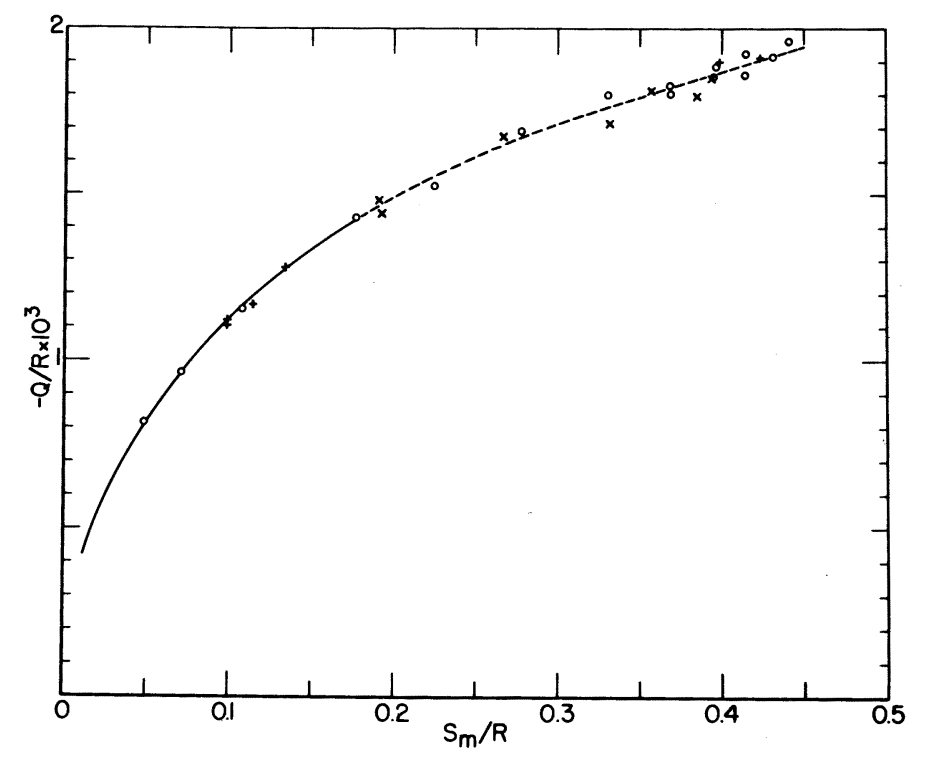

FIGURE 5

Heat content, $Q / R$, vs. entropy, $S_{m} / R(=\ln 2-S / R)$. - combination of linear portion of Fig. 3 and corresponding part of full line in Fig. 1. -..- least squares cubic equation fit to data points. 
We made use only of the linear portion of the master $Q$-curve (Fig. 3) and the corresponding portion of the $S-x$ curve of Fig. 1. There results the portion of the curve $0.01<S_{m} / R<0.18$, nd the individual demagnetization points are added there for completeness.

From 0.18 to the lowest entropies obtained we have plotted the individual points and fitted a curve through them and many points on the above mentioned curve. We used a computer "spline eit" [20] which determined "by least squares" two smoothly-joined cubic equations $(0.0144<$ $S_{m} / R<0.1042$ and $0.1042<S_{m} / R<0.4312$ ) and yielded a r.m.s. deviation of $2.045 \times 10^{-5}$ for $Q / R$ over the whole region.

Absolute temperatures were obtained by differentiating the curve of Fig. 5 , using the computed curve for the region $S_{m} / R>0.18$. (For that region only the r.m.s. deviation is $2.603 \times$ $10^{-5}$.) The results are shown as a plot of entropy versus Kelvin temperature in Fig. 6. The

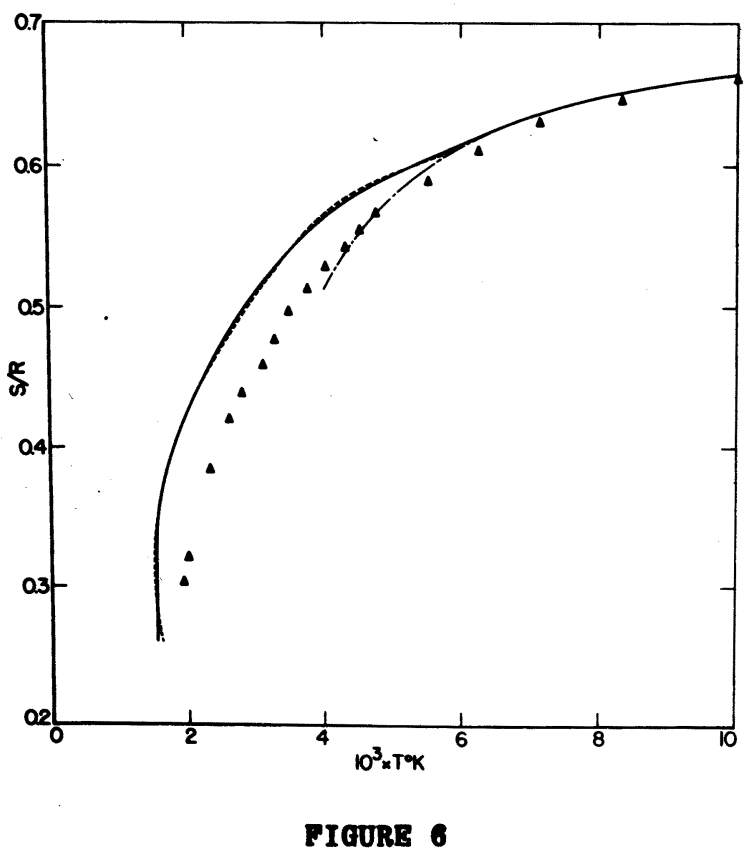

Entropy vs. Kelvin temperature. ----, from differentiation of curve in Fig. 5. - arbitrarily smoothed modification. $---_{-}$, from equation: ln $2-S / R=2.88 \times 10^{-6} T^{-2}$.

chain-curve delineates the equation ln $2-S / R=2.88 \times 10^{-6}, T^{-2}$ and the dashed curve the actual differentiation of Fig. 5. Having regard for the experimental uncertainties we deemed it appropriate to smooth out the curve by a relatively small redrawing (full line). The raw (dashed) curve curls back at the lowest entropies (which is not possible, physically) due to our inability to secure a curve for Fig. 5 without an inflexion point at low entropy. The substituted vertical portion is as satisfactory a representation of the data as any and, again, the possible additional error introduced thereby is negligible. The triangles included in Fig. 5 are from the data of Frankel, Shirley and Stone [8].

Table 1 summarizes our results with corresponding values of $H / T, S / R$, susceptibility, 
TABLE I

\begin{tabular}{|c|c|c|c|c|c|}
\hline $\begin{array}{l}10^{-3} \times H / T \\
\left(G \mathrm{deg}^{-1}\right)\end{array}$ & $g \beta H / k T$ & $S / R$ & $\begin{array}{c}10^{8} \times X^{1 / R} \\
\left(\operatorname{deg} G^{-2}\right)\end{array}$ & $\begin{array}{c}T \otimes \\
(m \text { deg })\end{array}$ & $\begin{array}{c}T \\
(m \text { deg })\end{array}$ \\
\hline 2.0 & 0.2472 & 0.6856 & 19.60 & 19.49 & 19.49 \\
\hline 2.5 & 0.3090 & 0.6814 & 24.45 & 15.63 & 15.63 \\
\hline 3.0 & 0.3708 & 0.6763 & 29.27 & 13.06 & 13.06 \\
\hline 3.5 & 0.4326 & 0.6703 & 34.04 & 11.23 & 11.23 \\
\hline 4.0 & 0.4944 & 0.6635 & 38.77 & 9.86 & 9.86 \\
\hline 4.5 & 0.5562 & 0.6559 & 43.45 & 8.80 & 8.80 \\
\hline 5.0 & 0.6180 & 0.6476 & 48.05 & 7.95 & 7.95 \\
\hline 5.5 & 0.6798 & 0.6386 & 52.61 & 7.26 & 7.26 \\
\hline 6.0 & 0.7416 & 0.6289 & 57.09 & 6.69 & 6.69 \\
\hline 6.5 & 0.8034 & 0.6185 & 61.49 & 6.21 & 6.18 \\
\hline 7.0 & 0.8652 & 0.6077 & 65.71 & 5.81 & 5.66 \\
\hline 7.5 & 0.9270 & 0.5963 & 69.73 & 5.48 & 5.14 \\
\hline 8 & 0.9888 & 0.5844 & 73.55 & 5.19 & 4.65 \\
\hline 9 & 1.1124 & 0.5595 & 80.38 & 4.75 & 3.90 \\
\hline 10 & 1.2360 & 0.5333 & 86.25 & 4.43 & 3.37 \\
\hline 11 & 1. 3596 & 0.5063 & 90.86 & 4.20 & 2.93 \\
\hline 12 & 1.4832 & 0.4788 & 94.70 & 4.03 & 2.56 \\
\hline 13 & 1.6068 & 0.4512 & 97.89 & 3.90 & 2.25 \\
\hline 15 & 1.8540 & 0.3965 & 102.41 & 3.73 & 1.78 \\
\hline 17 & 2.1012 & 0.3444 & 103. 76 & 3.68 & 1.54 \\
\hline 19 & 2.3484 & 0.2960 & 103.77 & 3.68 & 1.53 \\
\hline 21 & 2.5956 & 0.2521 & 103.52 & 3.69 & $(1.53)$ \\
\hline 23 & 2.8428 & 0.2131 & - & - & $(1.53)$ \\
\hline 25 & 3.0900 & 0.1790 & - & - & (1.53) \\
\hline
\end{tabular}

magnetic and Kelvin temperatures. Values of $\chi^{\prime} / R$ and $T \otimes$ for given $S / R$ were derived from curve $A$ of Fig. 1 and the straight line of Fig. 2. (One should recall that our experience indicates that demagnetizations for $S / R>0.67$, however, will not be isentropic.) Values of $T$ greater tha $0.0065^{\circ} \mathrm{K}$ were calculated from the relation $\ln 2-S / R=2.88 \times 10^{-6} T^{-2}$. Figures have been carried beyond the significant to facilitate faithful reproduction of the data, if desired.

Considering the case of a substance obeying Curie's law, $\mathrm{X}=c / T$, the Curie constant $c$ is given by

$$
c=N^{2} \beta^{2} S(S+1) / 3 k
$$

for a total number of spins $N$; the other symbols have their usual meaning.

Thus the quantity $c / R\left(=g^{2} \beta^{2} / 4 k^{2}\right.$ for $\left.S=1 / 2\right)$ is a simple one involving only fundamental constants and the pertinent $g$-value. The susceptibility is frequently expressed, therefore, as $\mathrm{x} / R(=c / R T)$ and may be calculated readily from relative susceptibility measurements, once the 
thermometer" has been calibrated in a region where the $T^{-1}$ dependence of $x$ obtains (equation 1)).

For CMN, $g_{\perp}$ (the appropriate $g$-value for these measurements) $=1.84[12]$ and $c / R=0.38_{2}$ $\mathrm{leg}^{2} \mathrm{G}^{-2}$. The susceptibility values in Fig. 1 were actually computed using $c / R=0.38$, but alues read from the curve $A$ have been adjusted to correspond to $0.38_{2}$ before compilation of olumn 4 in Table 1.

\section{Discussion}

\section{(a) Sources of error}

The method of deriving values for the absolute temperature used in this paper, i.e. from a unctional relationship between energy and entropy, relies upon one's ability to calibrate the ;amma ray heat source, which in turn requires the salt to exhibit a quadratic dependence of intropy upon temperature in the high regions of the magnetic thermometer's application.

The current observations demonstrate, with rising temperature, the expected behavior between 1.006 and $0.015^{\circ} \mathrm{K}$. At higher temperatures the effects of a small additional (anomalous) heat lapacity become manifest but the evidence is strongly in favor of this being non-magnetic in irigin (see Appendices). If this be so, then we can legitimately regard it as spurious for our urposes here, and rightfully adopt the procedures we have for obtaining corresponding $Q-x$ and i- $x$ relations valid for the spin system alone. (It is interesting to note that the highest ;emperature data shown (graphically) by Daniels and Robinson in their original paper [1] indiate a similar (but larger) anomalous heat capacity although their experimental scatter, it is irue, leaves room for doubt.)

The commonest errors arise from uncertainties in the entropy (from $H_{i}$ and $T_{i}$ ) and in the corresponding susceptibility (extrapolation of bridge reading to zero time); in the heatingiime measurement, including the effect of "drift" alone, prior to applying the gamme ray source; In the assumption that the combination of drift heat and gamma heat is constant for all measurelents; in fitting the respective straight lines to the data of Figs. 2 and 3 ; in deriving the ? $-S$ curve from the data points and in differentiating this. It is the latter which introduces is far the greatest uncertainty and an appreciation of this combined with an inspection of the source of the curve in Fig. 6 should convince one that derived temperatures between 3 and $5 \mathrm{~m}$. leg are most sensitive to the combined errors in measurement and data processing.

Having due regard for all the above factors we believe that the random error may be cogently issessed at $\pm 0.3 \mathrm{~m} \mathrm{deg}$ for all temperatures below $0.005^{\circ} \mathrm{K}$, diminishing rapidly to possible $0.1 \mathrm{~m} \mathrm{deg}$ at $0.006^{\circ} \mathrm{K}$. Above $0.0065^{\circ} \mathrm{K}$ and up to $4^{\circ} \mathrm{K}$, CMN should obey Curie's law exactly, as far as present measurement techniques can discern.

\section{(b) Comparison with other work}

The results reported here for our sphere II are in quite good accord with our previous, preliminary findings for sphere I and the "ellipsoid". They agree with the latter extremely closely lown to $0.003 \%$, below which they fall generally between the two earlier sets.

The measurements for sphere I are suspect because of the increased inhomogeneity of the 
heating for this large crystal. The high temperature reference point for the timing of the heating period was chosen too low, and insufficient data were taken in the (now known to be very significant) region of $0.02^{\circ} \mathrm{K}$ and above. Furthermore, the withdrawal of the cryostat from the magnet introduced some vibrational heating, leading to increased errors and scatter in the data.

The ellipsoid data were not taken primarily to obtain absolute temperatures and the data points are really too few for derived quantities to give rise to high confidence. Nevertheless the agreement between them and our current results is quite good.

We wish to emphasize, however, that, we do not consider that evidence exists for a shapedependent $S-T$ relation. Our sphere and "ellipsoid" data agree within the experimental uncertain ties which are, of course, quite large.

The experiments of Frankel, Shirley and Stone [8] using gamma-ray anisotropy as a thermometric parameter showed very close agreement between results obtained for specimens of differen shape. This approach should be very well suited to obtaining reliable data at the lowest temper tures, and where our method deteriorates. In the temperature region $0.0015-0.007^{\circ} \mathrm{K}$ (Fig. 6) our. temperatures are approximately $0.0005 \mathrm{deg}$ lower with a maximum separation of about $0.0007 \mathrm{deg}$ in the region $0.003-0.0045^{\circ} \mathrm{K}$.

Above $0.007^{\circ} \mathrm{K}$ Frankel et al. and we agree that $T=T T^{\otimes}$; our $S-T$ plots however lead to quite different estimates of $T$, given the entropy. This arises from our finding that $\ln 2-S / R=$ $2.88 \times 10^{-6} T^{-2}$ while they accept in $2-S / R=3.2 \times 10^{-6} T^{-2}$ as reported by Daniels and Robinson [1]. Actually they fitted their data to a theoretical curve above $0.006^{\circ} \mathrm{K}$ to derive a value for their quantity $B$, a hyperfine coupling parameter, and using only the fact that $T=T \otimes$ Then this value for $B$ was fed into the nuclear data obtained at very low temperatures to obtain estimates of the latter. Thus the elicitation of $B$ is seen to be of crucial importance in this method.

Between 0.006 and $0.0032^{\circ} \mathrm{K}$ the temperatures (at equivalent entropies) of reference [8] are slightly higher than those of reference [1]. (Below $0.0032^{\circ} \mathrm{K}$ the reverse situation sets in and the divergence increases rapidly.) This indication that the latter scale is quite good for representing the nuclear orientation data lends support to the corresponding $S-T$ relation. In addition. Frankel et al. state that "our $T^{*}$ " $\left(T \otimes\right.$ ?) "versus $(H / T)_{i}$ data agree well with those of Daniels and Robinson and were very reproducible using different crystals". The agreement is clearly depicted in a graphical presentation of the pertinent data [21].

This good agreement and the discrepancy with our data are puzzling. Figure 1 shows clearly that our $S-T \otimes$ results are very consistent between specimens of different shapes but quite different from those of Daniels and Robinson. And secondly one would expect the latter to become particular and unrepresentative of CMN in general for ln $2-S / R>0.2$, or $T<0.0033^{\circ} \mathrm{K}$, since the demagnetization correction is large in this region and would not be valid for a roughly rounded rectangular block as was actually used [22] by Daniels and Robinson.

Considering the probable error involved in extracting from the data a value for the slope of the line in Fig. 1, the difference between $2.88 \times 10^{-6}$ and $3.2 \times 10^{-6}$ is, in the context, enormous and presently inexplicable. While the oft-made assertion concerning the unique value of CMN in producing millidegree temperatures and in obeying Curie's law down to approximately $0.006^{\circ} \mathrm{K}$ remains valid, it is evident that much further study will be required to yield a full understanding of the low temperature properties of this substance. 


\section{F) Limitations of the CMN thermometer}

The present investigations relate to the properties of spheroidal-shape single crystals of N. This substance is, of course, highly anisotropic and hence the results, particularly at pe lowest temperatures, cannot be expected to apply to irregularly shaped crystals or aggreates of crystallites. This may be the reason for the marked discrepancy at the lowest temperaures between our conclusions and those of Abel, Anderson, Black and Wheatley [26], who conluded, using the properties of $\mathrm{He}^{3}$ as a temperature reference, that for powdered CMN $T^{*}=T$ $\mathrm{p}$ the lowest temperatures $\left(\sim 0.003^{\circ} \mathrm{K}\right)$.

The thermometric parameter being $x\left(\right.$ or $\left.T^{\otimes}\right)$, the salt diminishes in usefulness in the region here $x$ becomes an insensitive function of $S / R$. Inspection of Fig. 1 shows that the sensitivity iminishes rapidly for $\log _{\mathrm{e}} 2-S / R>0.28$, i. e. for $T<0.0019^{\circ} \mathrm{K}$ (from Fig. 6). In the liquid elium region, the thermometer is insensitive because of the smallness of $X$ and, further, the ighly advantageous feature of Curie law behavior disappears at an accelerating rate as $T$ rises bove, say, $5^{\circ} \mathrm{K}$ and the effect of energy doublets lying well above the ground state becomes ignificant $[5,6]$. Thus one may conclude that the $x-T$, or $T^{\otimes}-T$, relation for the $g_{\perp}$ direcion in spheroidal-shape single crystal specimens of CMN is a useful temperature scale for the ange $0.002-2^{\circ} \mathrm{K}$.

\section{References}

J.M. DANIELS and F.N.H. ROBINSON, Phil. Mag. 44, 630 (1953).

. R.P. HUDSON, in Temperature - Its Measurement and Control in Science and Industry, Vol. 3 , Part 1, p.51. Reinhold, New York (1962).

. R.P. HUDSON, R.S. KAESER and H.E. RADFORD, Proceedings of the 7 th International Conference on Low Temperature Physics, p.45. University of Toronto, Canada (1960).

C. B.P. FINN, R. ORBACH and W.P. WOLF, Proc. Phys. Soc. A77, 261 (1961); for a summary of different determinations of this splitting see reference [18].

3. R.P. HUDSON and W.R. HOSLER, Phys, Rev, 122, 1417 (1961).

3. M.J.M. LEASK, R. ORBACH, M.J.D. POTELL and W.P. MOLF, Proc. R. Soc. A272, 371 (1963).

p. F. CARBONI and R.C. SAPP, Ann. Phys. 33, 77 (1965).

3. R. B. FRANKEL, D.A. SHIRLEY and N.J. STONE, Phys, Rev. 140, A1020 (1965); 143, 334 (1966).

9. V.P. PESHKOV, $Z$. eksp. teor. Fiz. 48, 997 (1965); W.R. ABEL; A.C. ANDERSON, W.C. BLACK and J.C. MHEATLEY, Phys. Rev. Letters 16, 273 (1966).

10. N. KURTI and F. SIMON, Proc. R. Soc, A149, 161 (1935).

11. E. AMBLER and R.P. HUDSON, J. Chem. Phys. 27, 378 (1957).

12. A.H. COOKE, H.J. DUFFUS and W.P. MOLF, Phil. Mag. 44, 623 (1953).

13. This analysis was conducted in the Spectrochemistry Section, National Bureau of Standards. 
14. D. de KLERK and R.P. HUDSON, J. Res. Natl Bur. Std, 53, 173 (1954).

15. We differentiate between temperatures on the thermodynamic, or Kelvin, scale and on the "magnetic scale" by including and omitting the symbol $K$, respectively.

16. A. H. COOKE and R.A. HULL, Proc. R. Soc. A181, 83 (1942).

17. N. KURTI and F. SIMON, Phil. Mag. 26, 849 (1938).

18. C. A. BaIleY, Proc. Phys. Soc. 83, 369 (1964).

19. We consider a mole to contain $1 \mathrm{~g}$. ion of $\mathrm{Ce}^{3+}$, not two as indicated by the molecular formu and encountered in calorimetry papers.

20. Te are indebted to Mrs. CARLA MESSINA for making this program, a private communication fro Professor JOHN RICE, Purdue University, available to us.

21. R.B. FRANKEL, Thesis, University of California (unpublished).

22. J.M. DANIELS, private communication.

23. The analysis was carried out by Mrs. M.M. DARR and T.C. RAINS, Analytical Chemistry Divisio National Bureau of Standards.

24. Values for $b_{n}$ (hfs term in heat capacity "tail", $C_{n} / R=b_{n} T^{-2}$ ) were taken from A.H. CoOKE and H.J. DUFFuS, Proc. R. Soc. A229, 407 (1955); A.H. COOKE, H. MEYER and N.P. WOLF, Proc R. Soc. A237, 395 (1956).

25. The calorimetric measurements were made by J.H. COLWELL to whom the authors are indebted fol permission to include his results here.

26. T.R. ABEL, A.C. ANDERSON, W.C. BLACK and J.C. WHEATLEY, Physics 1, 337 (1965).

\section{APPENDIX 1}

\section{Quantitative analysis [23]}

The 3: 1 "ellipsoid" specimen was destructively tested. By atomic absorption spectrophotometry and comparison with standard solutions the $\mathrm{Cu}$ content was determined to be $1 \pm 0.3 \mu \mathrm{g} / \mathrm{g}$.

Cerium was separated from other rare earths by oxidation with AgO and extraction by di(2ethylhexyl) phosphoric acid in carbon tetrachloride. The rare earth fraction was precipitated with gallium as a carrier, ignited at $1000^{\circ} \mathrm{C}$, and the $\mathrm{Ga}_{2} \mathrm{O}_{3}$ fraction subjected to spectrochemical examination using synthetic reference standards. Radioactive tracers were employed to cross-check the separation procedure; Eu ${ }^{152,154}$ showed greater than 99 per cent recovery in the $\mathrm{Ga}_{2} \mathrm{O}_{3}$ and $\mathrm{Pr}^{144}$ was not extracted with the cerium.

The results, quoted in mass fraction of the CMN, were: 


$\begin{array}{lc}\text { Dy } & \text { not detected }\left(<10^{-6}\right) \\ \text { Gd } & \text { not } \text { detected }\left(<10^{-6}\right) \\ \text { La } & <5 \times 10^{-6} \\ \text { Lu } & <10^{-6} \\ \mathrm{Nd} & 3 \times 10^{-5} \\ \mathrm{Pr} & <5 \times 10^{-6} \\ \mathrm{Sm} & <5 \times 10^{-6} \\ \mathrm{~Tb} & \text { not detected }\left(<5 \times 10^{-6}\right) \\ \mathrm{Y} & \text { not detected }\left(<10^{-6}\right) \\ \mathrm{Yb} & <10^{-6}\end{array}$

\section{APPENDIX 2}

\section{Effect of Impurities}

From the foregoing Section we see that we need only consider the effects of Pr, Nd, and $\mathrm{Sm}$. These will contribute to the entropy via their hfs; abundances and relevant spins are $100 \%$, $5 / 2 ; 20 \%, 7 / 2$; and $29 \%, 7 / 2$ respectively. As the mass fraction of the $\mathrm{Ce}^{3+}$ itself is approximately 0.18 , the effective concentrations, relative to their contributing additional entropy, are: $<3 \times 10^{-5}, 1.7 \times 10^{-4}$ and $<3 \times 10^{-5}$ respectively.

Thus for the contributions to $S / R$ we calculate:

Pr: (less than) $1 \times 3 \times 10^{-5} \times \ln 6=<5.4 \times 10^{-5}$

Nd:

$$
0.20 \times 1.7 \times 10^{-4} \times \ln 8=7.1 \times 10^{-5}
$$

$\mathrm{Sm}$ : (less than) $0.29 \times 3 \times 10^{-5} \times \ln 8=<1.8=10^{-5}$

and, in total, < $1.4 \times 10^{-4}$.

Failure to include such a contribution to the entropies calculated for the points in Figure 1 would obviously not modify the derived value for the slope of $2.88 \times 10^{-6}$.

These impurities would, however, add a contribution to the b-value (heat capacity coefficient of $T^{-2}$ ) in the liquid helium region [24]. This appears in the expression for the entropy as $b / 2$; we found [3] $3.15 \times 10^{-6}$ instead of the figure $2.88 \times 10^{-6}$ but the impurity contribution of $0.15 \times 10^{-6}$ (mostly due to $\mathrm{Pr}$ ) accounts for only one half of the difference. The rest can readily be accounted for by imprecision in the paramagnetic relaxation determination. 


\section{APPENDIX 3}

\section{Anomalous heat capacity}

Referring to Fig. 4, for any given value of $n^{\prime}$ (or $T^{\otimes}$ ) the slope of the curve and the slop of the "ideal" straight line are in inverse ratio to that of the total heat capacity and the dipole-dipole heat capacity $5.76 \times 10^{-6} \mathrm{~T}^{-2}$. The total heat capacity may therefore be derived.

Again a spline fit was used to place a "least squares" curve through the points (actually w fitted to $500-n^{\prime}=n^{\prime \prime}$ ), two cubic equations giving an excellent fit with a r.m.s. deviation in $n$ " of 1.5356. The curve was differentiated algebraically and the computation made of total heat capacity, then the anomalous fraction by subtraction.

The results are shown in Fig. 7. We emphasize that the numerical values diminish in validits

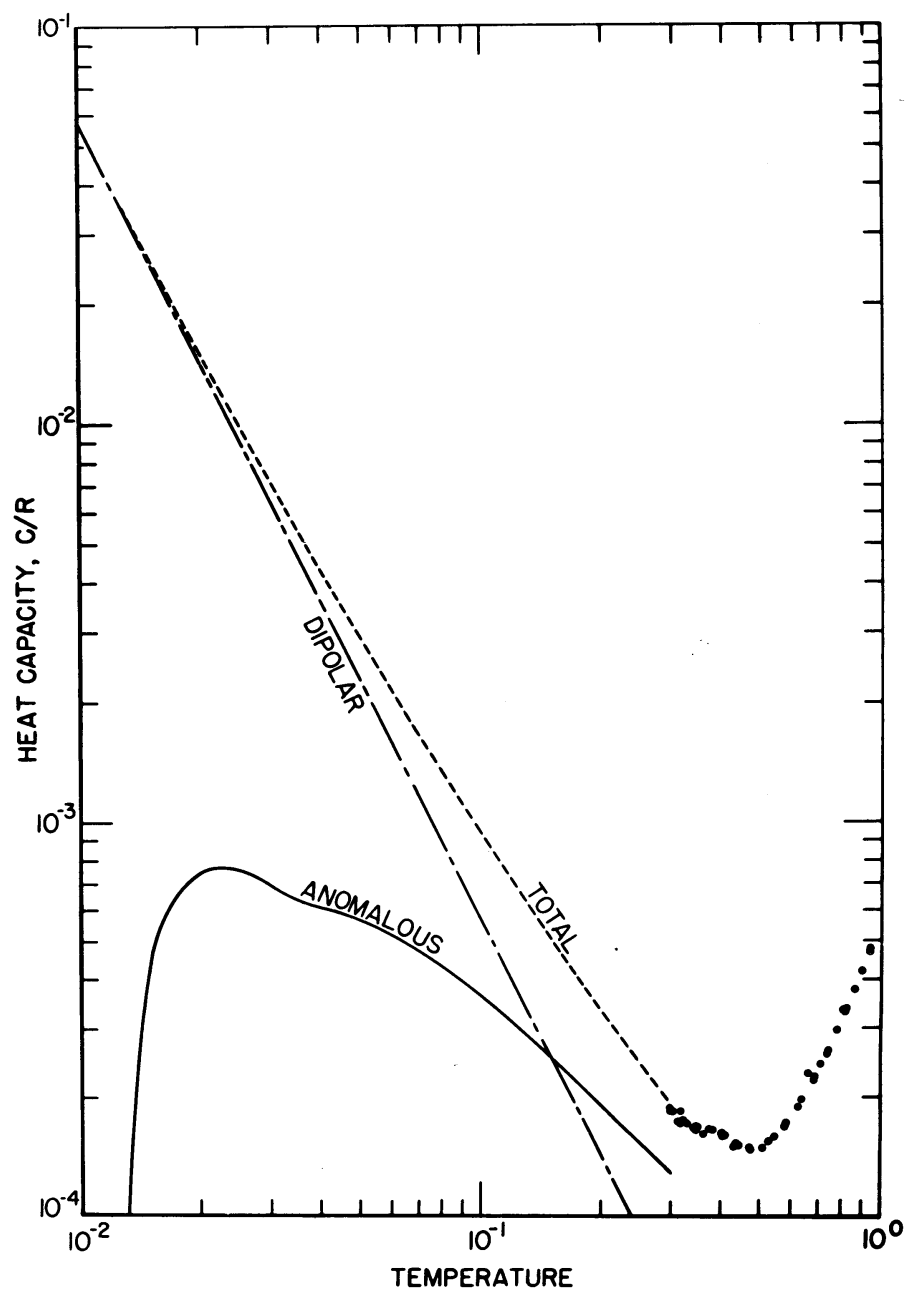

FIGURE 7

Heat capacity vs. temperature. Curve labelled "dipolar" is $5.76 \times 10^{-6} \mathrm{~T}^{-2}$.

Points in lower right-hand corner due to Colwell [25]. 
th the temperature extremes: at the low end because we are taking the small difference between wo large quantities, and at the high end because the primary experimental data are too sparse to determine the curve accurately. The general features of Fig. 7 are significant, however, and these show a peak in the anomaly around $0.02^{\circ} \mathrm{K}$, and a high temperature "tail" which varies as $\mathrm{r}^{-1}$ and in the region of $0.3^{\circ} \mathrm{K}$ is much greater than the dipolar contribution. Also plotted are the results of conventional calorimetric determinations of the total heat capacity of a large single crystal of CMN [25] which appear to agree remarkably well with our derived data (though the measurements only meet, i.e. do not overlap).

These latter data cannot be represented by a combination of two terms varying respectively as $T^{-2}$ and $T^{3}$. They suggest notable departures from $T^{3}$ below $1^{\circ} \mathrm{K}$ and in the region where any magnetic contribution would be negligible. (The measurements when carried to still higher temperatures agree well with the findings of Bailey [18] for the region above $2^{\circ} \mathrm{K}$.)

The entropy associated with the anomaly curve of Fig. 7 is $1.4 \times 10^{-3} R$ approximately, or ten times as large as that arising from the hfs of the impurities (Appendix 2). On the other hand, it is still a very small quantity. In magnitude and temperature range it is highly suggestive of a magnetic impurity, but we have shown that this cannot be substantiated; a nonmagnetic source, and in poor thermal contact with the spin system, is more likely. It is manifest in all our specimens but their varying shape, size, and mounting rule out the possibility of its arising from surface coating or mechanical support. It would be interesting to conduct low temperature heat capacity measurements on the (very pure) lanthanum double nitrate, and we plan to do this in the near future. 